 \\ Clinical Studies and Medical Case Reports
}

\section{Case Report of Pancreas Divi- sum and Main Duct Intraductal Papillary Mucinous Neoplasm of the Pancreas}

\author{
Pauline Joy F Santos ${ }^{1 *}$, David K Imagawa ${ }^{1}$, Chandana Lall ${ }^{2}$ \\ and John G Lee ${ }^{3}$
}

${ }^{1}$ Division of Hepatobiliary and Pancreas Surgery/lslet Cell Transplantation, Department of Surgery, University of California, Irvine Medical Center, USA

${ }^{2}$ Department of Radiology, University of California, Irvine Medical Center, USA

${ }^{3}$ Department of Medicine, University of California, Irvine Medical Center, USA

\begin{abstract}
Pancreas Divisum (PD) is the most common congenital anomaly of the pancreas. It is well known that PD causes pancreatitis and is associated with pancreatic neoplasms. However, its association with Intraductal Papillary Mucinous Neoplasms (IPMN) is rare, and its association with main duct IPMN is even more rare. Our case of PD and main duct IPMN is a rare and interesting finding. The images from MRI and EUS/ERCP nicely delineate the PD anatomy and IPMN pathology.
\end{abstract}

\section{Introduction}

Pancreas Divisum (PD) is the most common congenital anomaly of the pancreas. It results from the failure of fusion of the dorsal and ventral anlage. Its prevalence in the general population is not well-established, but data suggests it is comparable to idiopathic pancreatitis [1]. PD prevalence may have geographic variability given the reported incidence of PD being 5 to $10 \%$ in Western countries and 1 to $2 \%$ in Asia [2]. PD has been shown to be associated with pancreatitis and pancreatic neoplasms. In particular, PD has been associated with pancreatic tumor development, most commonly ductal carcinomas. Few PDs are associated with Intraductal Papillary Mucinous Neoplasms (IPMN) [2]. Furthermore, PD with main duct IPMN is even more rare [3].

IPMN is caused by mucin-producing neoplastic epithelia and can present as either a cystic or saccular dilation of the branch duct or

*Corresponding author: Pauline Joy F Santos, Division of Hepatobiliary and Pancreas Surgery/lslet Cell Transplantation, Department of Surgery, University of California, Irvine Medical Center, USA, Tel: +1 7144563884; Fax: +1 7144563487; E-mail: pjsantos@uci.edu

Citation: Santos PJF, Imagawa DK, Lall C, Lee JG (2016) Case Report of Pancreas Divisum and Main Duct Intraductal Papillary Mucinous Neoplasm of the Pancreas. J Clin Stud Med Case Rep 3: 033.

Received: March 08, 2016; Accepted: May 20, 2016; Published: June 03, 2016 main duct of the pancreas [4]. IPMN occurs in the sixth and seventh decades of life, and affects men more than women [5]. The ratio of main duct IPMN in males to females has been reported from 1.1 to 3:1. The true incidence of IPMN is not known because it is usually small and asymptomatic [6]. We present a case of main duct IPMN and PD.

\section{Case Report}

A 57-year-old female with history of dysphagia, alcohol abuse, and gallstone pancreatitis status post cholecystectomy 4 years ago was transferred for evaluation of cholangitis. IPMN was seen on EUS/ERCP (Figures 1, 2). EUS/ERCP also demonstrated a common bile duct measuring $23 \mathrm{~mm}$, pancreas divisum with $10 \mathrm{~mm}$ duct of Santorini, and a transient mucin globule. The mucin ball is also seen floating in the pancreatic duct (Video clip). Pancreatic juice aspiration demonstrated atypical glandular cells and mucin compatible with IPMN. The atypical glandular cells show features of low-moderate dysplasia. On MRI, pancreas divisum is evident, as well as a dilated duct (Figures 3,4$)$. Patient is not a surgical candidate given her liver disease and was instead observed. She was recommended to abstain from alcohol and reduce narcotic pain medication. She will be reevaluated in 2 to 3 months with nuclear medicine liver SPECT scan.

\section{Video Clip}

http://www.heraldopenaccess.us/fulltext/Clinical-Studies-\&-Medical-Case-Reports/Sample.php

Pancreatoscopy showing floating mucin in the pancreatic duct and the whitish mucin globule

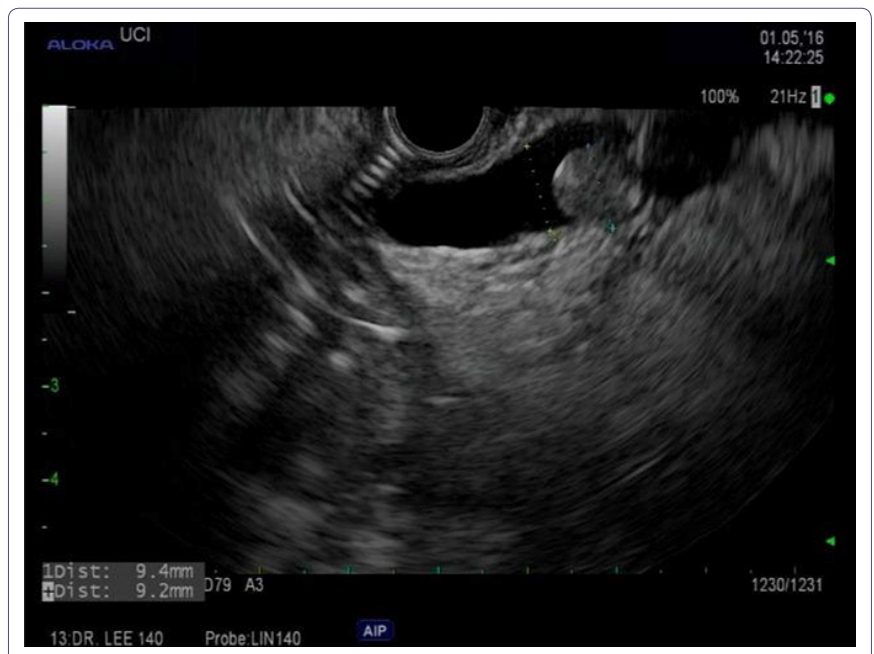

Figure 1: EUS showed dilated pancreatic duct of Santorini (main duct) with mucin ball near the minor papilla.

\section{Discussion}

IPMN of the pancreas include features of ductal dilation, intraductal papillary growth, and thick mucus secretion. They develop from the pancreatic duct epithelium in the main pancreatic duct or its branches and have malignant potential. IPMN accounts for $7.5 \%$ of clinically diagnosed pancreatic neoplasms and $16.3-25 \%$ of 


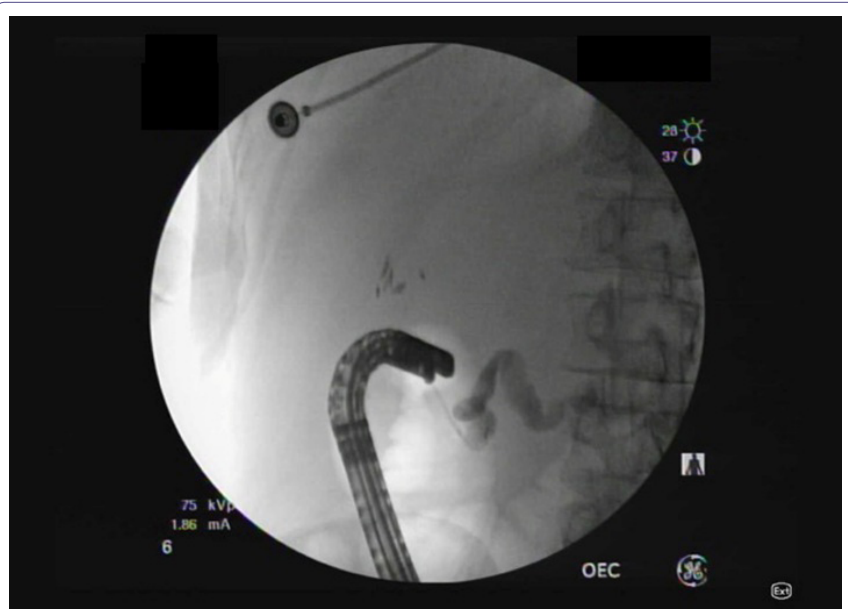

Figure 2: ERCP performed through the minor papilla showed pancreas divisum. Duodenoscope is in long position for the minor papilla cannulation which was done showing dilated main pancreatic duct consistent with pancreas divisum and main duct IPMN.

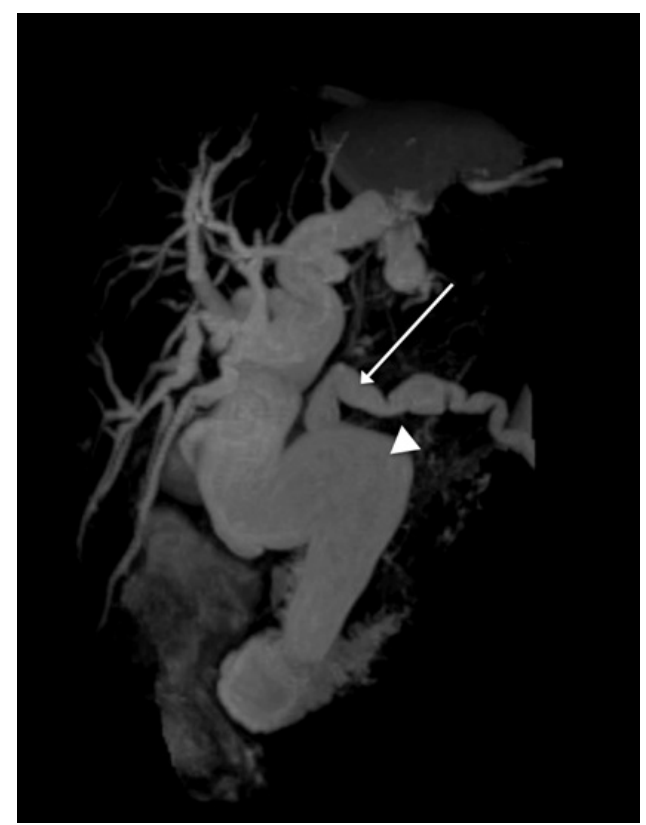

Figure 3: MRI image showing dilated beaded pancreas divisum (long arrow) and common bile duct (arrowhead).

surgically resected pancreatic neoplasms [7]. The risk of malignancy for main duct IPMN is significant, ranging from $57 \%$ to $92 \%$ compared to branch duct IPMN, which has a risk of malignancy from $6 \%$ to $46 \%$ [7].

PD has been seen with pancreatitis and pancreatic neoplasms, but rarely with IPMN. In a retrospective single-center study, only $5 \%$ of patients with PD had IPMN [8]. In a recent case report and review of the literature, only 16 cases of IPMN associated with pancreas divisum, including our case, were identified. More than half of the patients reported had branch-duct type IPMN [2].

Main duct IPMN is associated with increased risk of malignant transformation and dilation of $>10 \mathrm{~mm}$ has an even higher risk of malignancy [7]. In this report, the IPMN is in the main pancreatic duct and measures $10 \mathrm{~mm}$ and therefore, this patient has increased risk of malignant transformation. Surgical intervention is the

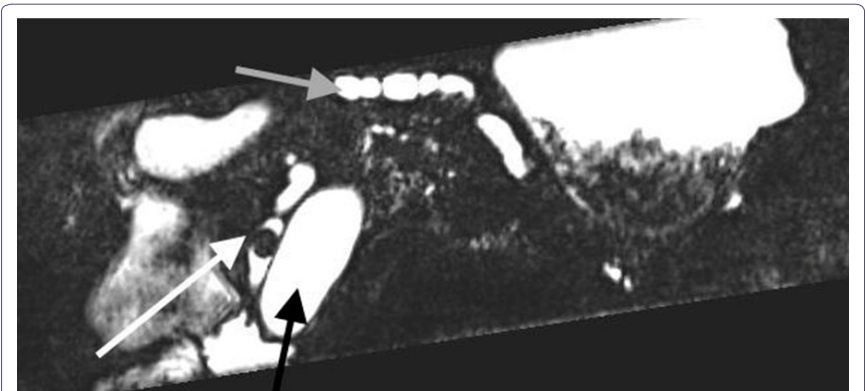

Figure 4: MRI image showing dilated beaded pancreas divisum with luminal mucin ball (white arrow), dilated beaded pancreas divisum (gray arrow), and dilated common bile duct (black arrow).

management of IPMN because of malignant potential. For main duct IPMN specifically, total pancreatectomy is the procedure of choice. This also requires an oncological lymphadenectomy, which compromises the lymph nodes of the hepatoduodenal ligament and surrounding lymph nodes of the celiac axis and superior mesenteric artery. Additionally, reconstruction includes pancreaticojejunostomy or pancreaticogastrostomy, hepaticojejunostomy, and duodeno- or gastrojejunostomy [9]. However, given the patient's cirrhotic morphology, surgery was not recommended because of the potential for liver failure.

IPMN accounts for $5.1 \%$ of pancreatic carcinomas in PD [8]. The mechanism may involve increased incidence of chronic pancreatitis in the setting of IPMN [10]. However, the relationship between IPMN and PD is unclear. Given the patient's complicated history, the implications of PD and main duct IPMN in her past presentation of pancreatitis are uncertain. However, this case demonstrates the importance of familiarity with this anatomic variation and recognition of IPMN on imaging for appropriate management and prevention of malignant transformation.

\section{Conclusion}

Recognition of PD and its potential complications are critical in appropriate management of patients presenting with a history of pancreatitis. Although the relationship between PD and IPMN is unclear, this case supports the importance of further elucidating the mechanism by which IPMN develops in the setting of PD.

\section{References}

1. DiMagno MJ, Wamsteker EJ (2011) Pancreas divisum. Curr Gastroenterol Rep 13: 150-156

2. Nishi T, Kawabata Y, Ishikawa N, Araki A, Yano S, et al. (2015) Intraducta papillary mucinous carcinoma of the pancreas associated with pancreas divisum: a case report and review of the literature. BMC Gastroenterology 15: 78.

3. Takuma K, Kamisawa T, Tabata T, Egawa N, Igarashi Y (2010) Pancreatic diseases associated with pancreas divisum. Dig Surg 27: 144-148.

4. Tanaka M (2015) International consensus on the management of intraductal papillary mucinous neoplasm of the pancreas. Ann Transl Med 3: 286

5. Castellano-Megías VM, Andrés Cl, López-Alonso G, Colina-Ruizdelgado F (2014) Pathological features and diagnosis of intraductal papillary mucinous neoplasm of the pancreas. World J Gastrointest Oncol 6: 311-324.

6. Konstantinou F, Syrigos KN, Saif MW (2013) Intraductal Papillary Mucinous Neoplasms of the pancreas (IPMNs): epidemiology, diagnosis and future aspects. JOP 14: 141-144.

7. Norman Oneil Machado, Hani al Qadhi, Khalifa al Wahibi (2015) Intraducta Papillary Mucinous Neoplasm of Pancreas. N Am J Med Sci. 7: 160-175.

8. Nishino T, Toki F, Oi I, Oyama H, Hatori T, et al. (2006) Prevalence of pancreatic and biliary tract tumors in pancreas divisum. J Gastroenterol 41: 10881093. 
Citation: Santos PJF, Imagawa DK, Lall C, Lee JG (2016) Case Report of Pancreas Divisum and Main Duct Intraductal Papillary Mucinous Neoplasm of the Pancreas. J Clin Stud Med Case Rep 3: 033.

- Page 3 of $3 \cdot$

9. Hackert T, Fritz S, Büchler MW (2015) Main- and Branch-Duct Intraductal Papillary Mucinous Neoplasms: Extent of Surgical Resection. Viszeralmedizin 31: $38-42$.
10. Talamini G, Zamboni G, Salvia R, Capelli P, Sartori N, et al. (2006) Intraductal papillary mucinous neoplasms and chronic pancreatitis. Pancreatology 6 : $626-634$ 
Citation: Santos PJF, Imagawa DK, Lall C, Lee JG (2016) Case Report of Pancreas Divisum and Main Duct Intraductal Papillary Mucinous Neoplasm of the Pancreas. J Clin Stud Med Case Rep 3: 033.

- Page 4 of $3 \cdot$ 